\title{
Understanding the aging lexicon by linking individuals' experience, semantic networks, and cognitive performance
}

\author{
Dirk U. Wulff ${ }^{1,2}$, Simon De Deyne ${ }^{3}$, Samuel Aeschbach ${ }^{1}$, and Rui Mata ${ }^{1,2}$ \\ ${ }^{1}$ University of Basel \\ ${ }^{2}$ Max Planck Institute for Human Development \\ ${ }^{3}$ University of Melbourne
}

\begin{abstract}
People undergo many idiosyncratic experiences throughout their lives that may contribute to individual differences in the size and structure of their knowledge representations. Ultimately, these can have important implications for individuals' cognitive performance. We review evidence that suggests a relationship between individual experiences, the size and structure of semantic representations, as well as individual and age differences in cognitive performance. We conclude that the extent to which experience-dependent changes in semantic representations contribute to individual differences in cognitive aging remains unclear. To help fill this gap, we outline an empirical agenda involving the concurrent assessment of large-scale semantic networks and cognitive performance in younger and older adults, and present preliminary data to establish the feasibility and limitations of such empirical approaches.
\end{abstract}

Keywords: semantic networks, cognitive aging, individual differences

From childhood and adolescence onwards, the average human reads a couple of books each year, watches hundreds of hours of TV, and spends many hours on social media, leading to the accumulation of a large and, potentially, largely unique, set of experiences during a lifetime (Twenge et al., 2019). To what extent does the accumulation of such idiosyncratic experiences contribute to individual differences in thought and judgment across the life span?

Aging research has long realized the importance not only of describing the modal changes in cognition across the life span but also that "cognitive development in adulthood and old age differs substantially from person to person and is malleable within individuals" (Lindenberger, 2014). Despite the field's direct acknowledgment of inter-individual differences, we still know little about the sources of such differences and the extent to which idiosyncratic life experiences contribute to cognitive performance. Crucially, some voices have raised the possibility that cumulative experience is a major factor

Dirk U. Wulff (D) https://orcid.org/0000-0002-4008-8022 Simon De Deyne (D) https://orcid.org/0000-0002-7899-6210 Rui Mata (D) https://orcid.org/0000-0002-1679-906X

We are grateful to Laura Wiles for editing the manuscript. This work was supported by a grant from the Swiss Science Foundation (100015_197315) to Dirk U. Wulff.

Correspondence concerning this article should be addressed to Dirk U. Wulff, Department of Psychology, University of Basel, Missionsstrasse 60-62, 4055 Basel, Switzerland. E-mail: dirk.wulff@gmail.com underlying typical age-related patterns, such as decreased memory performance with increased age (Buchler \& Reder, 2007, Ramscar et al., 2014).

This article contributes to this effort by reviewing evidence on the effects of cumulative experience on cognition. We conclude that the existing literature has not fully established the links between experience, cognitive representation, and cognitive performance. One central limit of past work has been a lack of direct assessment of individuals' mental representations. As a result, we propose an empirical agenda to fill this gap and present a study that illustrates the feasibility and limitations of our approach, which aims to elicit large-scale semantic networks from single individuals. Finally, we discuss the limitations and implications of the outlined research agenda to understand individual and age differences.

\section{From Experience to Cognitive Performance: An Overview of the Current Literature}

In this section, we assess the current state of the psychological literature that has investigated the links between environmental exposure, the size and structure of semantic representations, and cognitive performance across the life span (see Figure 1). Our goal is both to provide a brief overview of the current literature and to highlight the main gaps that must be filled to answer the question above concerning the role of individual experience in cognitive representations and performance.

There are several lines of research supporting a close link between cumulative experience and cognitive performance 


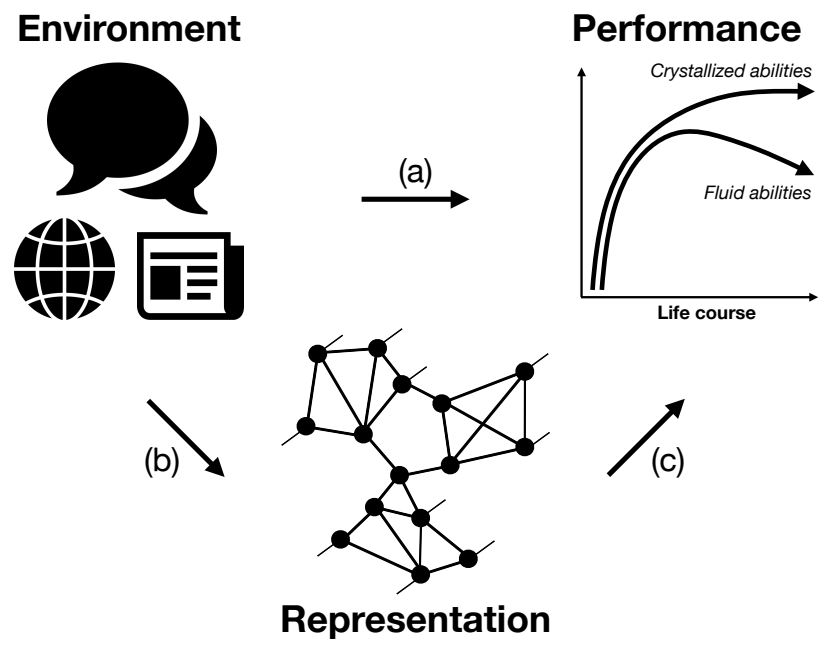

Figure 1

Research pathways that have linked environment, representation, and cognitive performance across the lifespan. Pathway (a) represents research studying the association between the environment and cognitive performance without explicit consideration of the underlying mental representations. Pathway (b) represents research on the association between environmental exposure and mental representations. Pathway (c) represents research on the association between mental representations and cognitive performance.

(see Figure 1, path a: Environment-Performance), of which three stand out. First, research that summarizes the association between print exposure and reading performance in children and young adults underlines the tremendous impact of experience on linguistic proficiency. Specifically, metaanalytic results show moderate to strong correlations between print exposure and reading comprehension or spelling (Mol \& Bus, 2011). This work supports the idea of an "upward spiral", such that individuals who are more exposed to written text become more proficient in reading and comprehension, which, in turn, leads to increased print exposure, further increasing linguistic proficiency. Second, one of the best-documented findings in aging research concerns the observed increases in crystallized abilities across the life span, as reflected in vocabulary size (Verhaeghen, 2003, for a metaanalysis). This work suggests that even as adults, individuals continue to expand their vocabulary store throughout their lives (Brysbaert et al., 2016), which is compatible with the idea that such vocabulary growth is related to individuals' lifetime formal or informal education. Notably, such increases in knowledge seem to have general implications for cognitive performance. For example, older adults are more likely to rely on prediction in reading because of their additional reading experience (Huettig \& Pickering, 2019). Third, research on expertise suggests that increased cumulative experience leads to domain-specific memory perfor- mance (Sala \& Gobet, 2017, for a meta-analysis), such as, for instance, experienced chess players being able to memorize both realistic and random chess position better than novices (Gobet \& Simon, 1996). All in all, such findings coalesce to make a strong case for the importance of environmental effects on cognitive performance and suggest that these effects are cumulative and can be domain-specific.

Most, if not all, psychologists will find it trivial to state that the effects of cumulative experience are somehow mediated by its effects on mental representations and processes (Figure 1, path b, Environment-Representation). Despite the truism and the increasing consensus that the lexical-semantic space continues to be shaped by personal linguistic experience throughout the life span (Rodd, 2020), only recently have researchers started to probe more deeply into the effects of cumulative experience on the structure of knowledge representations. Some researchers have adopted graphbased approaches to capture potential structural changes to the mental lexicon that occur across the life span as a function of experience (Wulff et al., 2019). The current literature suggests that cumulative experience has implications for not only the size but also the structure of mental representations (Dubossarsky et al., 2017). For example, Dubossarsky and colleagues conducted a network analysis of free association data from thousands of individuals (10 to 84 years of age), and found older adults' semantic networks were less connected (i.e., the words in the network have lower average degrees), less organized (i.e., the words in the network have a lower average local clustering coefficient), and less efficient (i.e., the shortest path length between any two words in the network is greater) relative to those of younger adults. Crucially, new methods are becoming available that promise ever greater insight and precision in mapping individual and group differences in the structure of mental representations (Morais et al., 2013; Zemla \& Austerweil, 2018). Table 1 presents an overview of a few approaches to measuring individual-level mental representations and we discuss in more detail pros and cons of each approach in the next section (cf. "An Empirical Agenda").

Finally, research is accumulating that establishes direct links between the structure of semantic representations and cognitive performance (Figure 1, path c, RepresentationPerformance). For example, various studies on memory recall show impaired performance when words represented as nodes within a semantic network have lower clustering (Nelson et al., 1993). More recent work has expanded this line of research to understand how lexical and semantic structure is crucial to individual cognitive performance in various domains, such as intelligence and creativity (Kenett \& Faust, 2019, Li et al., 2020). For a review, see Wulff et al. (2019). These more recent studies are particularly relevant because they have started relying on individual or small group estimates of mental representations and how macro-properties 
Table 1

Approaches to measuring individual-level semantic networks

\begin{tabular}{|c|c|c|c|c|}
\hline Paradigm & Description & Scope & Comparability & References \\
\hline Relatedness judgments & $\begin{array}{l}\text { Individuals rate the relation, i.e., sim- } \\
\text { ilarity, between pairs of items }\end{array}$ & Limited & High & $\begin{array}{l}\text { (Benedek et al., 2017 } \\
\text { Roads \& Love, 2020) }\end{array}$ \\
\hline $\begin{array}{l}\text { Free association (snow- } \\
\text { ball) }\end{array}$ & $\begin{array}{l}\text { Individuals generate one or more as- } \\
\text { sociations to word cues, which are } \\
\text { participant generated }\end{array}$ & Broad & Low & (Morais et al., 2013) \\
\hline $\begin{array}{l}\text { Free association (fixed } \\
\text { list) }\end{array}$ & $\begin{array}{l}\text { Individuals generate one or more as- } \\
\text { sociations to experimenter-generated } \\
\text { word cues }\end{array}$ & Broad & High & see below \\
\hline
\end{tabular}

Note. Scope $=$ Ability of the paradigm to provide coverage of a large set of semantic categories; Comparability = Ability of the paradigm to provide comparable coverage of semantic representation from different individuals.

of representational networks impact cognitive performance. However, one should note that this work has not established a direct link between an individual's cumulative experience and the size or structure of the mental representations.

All in all, these different lines of research support the idea that cumulative personal experience plays a crucial role in determining individual differences in cognitive performance across the life span. However, much of this work is correlational (Nation, 2017, for a similar critique) and not carried out at the level of the individual. As a result, the mechanisms that tie cumulative exposure to performance are still under investigation. In what follows, we propose that to truly understand the consequences of individual experience on mental representation and cognitive performance, the concurrent assessment of individuals' unique environments, as well as their mental representations and cognitive performance, is needed.

\section{An Empirical Agenda}

As outlined above, the current literature has made large strides towards understanding the components linking cumulative experience to cognitive performance: We know that younger and older adults differ in the amounts and kinds of experiences made, the contents and structure of mental representations, and that there are systematic age and individual differences in both fluid and crystallized performance. However, presently, we cannot confidently estimate the portion of individual differences in performance across the life span due to the accumulation of specific types of experience. What is missing, in our mind, is a concerted empirical agenda that investigates the Environment-Representation and the Representation-Performance pathways at the level of the individual, thus allowing us to quantify how a certain type or quantity of experience translates into the size or structure of cognitive representations, and, ultimately, into differences in cognitive performance. This goal presents challenges on several levels, and we discuss two major ones below: first, the challenge of measuring individuals' environments, and, second, the challenge of capturing the content and structure of single individuals' mental representations.

Concerning the challenge of measuring individuals' environments, one main limiting factor is currently the lack of available data. Put simply, the field lacks context-aware longitudinal projects that provide a characterization of the environments experienced by individuals over time. Although unprecedented, large amounts of contextualized text and speech data are now available to scientists (e.g., Love et al., 2017, Schröter \& Schroeder, 2017), few of these data sets present data on the level of the individual or distinguish between age groups. Crucially, any individual's environment consists of not only linguistic information, but also rich multimodal sensorial information (e.g., De Deyne et al., 2021). Thus, a major challenge for future research is to create individual-annotated, multimodal corpora accessible for research. There is optimism in the field that the rise of the quantified-self movement and the availability of new "digital tracing" methods can provide multiple data streams to feed computational modeling efforts that use these data to create models of individual's mental representations (Wulff et al., 2019). These models would be crucial to generate expectations about the actual estimates of individuals' mental representations.

Concerning the challenge of mapping individuals' mental representations, and as discussed above, there are now different approaches to investigating the size and structure of mental representations (see Table 1). However, not all techniques are equally suited to uncover the full breadth of a single individual's mental representation (scope) while ensuring com- 
parability between individuals in the lexical-semantic space covered (comparability). For example, verbal fluency tasks are limited to specific categories (e.g., animals) and the structure of a single semantic category may not be representative of the entire semantic network (De Deyne \& Storms, 2008). In addition, learning about within-category structure is not very informative about the links between categories. In turn, other methods, such as relatedness judgments, can be powerful in providing comparability between individuals because participants can be presented with the same pairs of concepts. However, asking individuals to provide ratings to all possible pairs becomes prohibitively demanding with even small sets of stimuli because this implies thousands of pairedcomparisons (Wulff et al., 2018, October 29 Compared to other methods, free associations are relatively economical and can provide high scope. However, free-association methods that ask individuals to generate associations in a snowball method (Morais et al., 2013) may reduce comparability across participants due to path-dependency in any individual's search of the representational store. In comparison, free associations with fixed, experimenter-generated lists may provide a better choice in both scope and comparability. One should note that free association has also been the method of choice for most previous large-scale assessments of aggregate semantic networks (De Deyne \& Storms, 2008, Steyvers et al., 2004).

All in all, our review of the literature, as well as assessment of the challenges above, highlights several future directions: first, providing a better description of the idiosyncratic experiences of individuals that can inform computational and learning models of linguistic and semantic cognition, and second, mapping the mental representations of single individuals that can later be matched to expectations about the role of experience in cognitive performance and the potential consequences. In what follows, we provide our own attempt at tackling the second challenge of mapping individual semantic networks and linking them to cognitive performance.

\section{An example study for the Representation-Performance pathway: MySWOW}

We designed and piloted the My Small World of Words (MySWOW) study to assess the feasibility of mapping individual semantic networks from word associations and analyzing how they are linked to cognitive performance.

The procedure of our MySWOW study was inspired by ongoing efforts to obtain word association norms for several languages in a large online citizen-science project, the Small World of Words (SWOW) study (https://smallworldofwords. org). The project currently includes data from over half a million participants in 15 languages. So far, this project has resulted in a set of useful linguistic resources for both Dutch (De Deyne et al., 2012) and English (De Deyne et al., 2018) and will release additional resources for other languages including German (https://smallworldofwords.org/de) in the near future. The adoption of the same procedure for the large, population-based SWOW and the smaller study of individuals, MySWOW, promises future assessments of the comparability between the results from aggregate networks and those of single individuals or groups (e.g., younger, older).

We largely adopted the same procedure used in SWOW, which asks participants to provide three associates to a given cue (e.g., "cat"). However, SWOW typically presents volunteers with 18 cues that provide responses in the course of minutes. In contrast, we asked each individual participant to provide answers to thousands of cues over the course of weeks. Specifically, each participants in our study was asked to provide three associations to a total of 3,000 unique cues and 600 repeated cues, resulting in a total of 10,800 responses per participant. The repeated cues were included in order to assess the consistency of participants' associations.

We obtained data for four younger (aged 24 to 28) and four older (aged 68 to 70) native German speakers. In an initial session, participants were briefed in person and received instructions concerning the dedicated online tool that they could use to complete the word associations. Participants then completed the word associations from home over the course of 2-4 weeks. After finishing the word-association task, participants returned to the laboratory for cognitive testing on several memory and linguistic tasks. In particular, we focused on verbal fluency, paired associated, and episodic memory tasks that have often been used to estimate and understand individual and age differences in cognitive performance (Ramscar et al., 2017; Zemla \& Austerweil, 2018

\section{Differences in the semantic networks of younger and older individuals}

In what follows, we document our efforts to obtain individual semantic networks from word association data in MySWOW and report a qualitative comparison of the results with those from past work, which suggests structural differences between the semantic representations of younger and older adults (Dubossarsky et al., 2017, Wulff et al., 2019).

For each individual, we created uni-partite networks by placing weighted edges between responses and corresponding cues. This resulted in eight individual networks containing 4,836 to 6,461 nodes, which is comparable in size to previously obtained large-scale aggregate associative networks (Morais et al., 2013; Steyvers et al., 2004). Figure 2 illustrates the network of one individual, highlighting the

\footnotetext{
${ }^{1}$ A recent alternative approach (Roads \& Love, 2020) that relies on ranking and active learning algorithms can provide a solution to the problem of scale.

${ }^{2}$ We provide a full account of our design, participants, and methods in a companion data paper https://www.overleaf.com/read/ bdqyfgzsrgpr)
} 
most central words according to PageRank and the underlying structure by identifying clusters extracted using the Louvain method (Blondel et al., 2008).

Networks strongly overlapped in content with each other and with existing SWOW data sets. Table 2 presents the ten most central words according to node degree for each of the individual networks. Words in bold are also among the most central words in either the English (De Deyne et al., 2019) or the German ${ }^{3}$ SWOW data. Considering only the 2,111 words shared between all individual networks, the degree distributions of individual networks showed average correlations of $r=.57$. Consistent with earlier work suggesting a progression of network differentiation with age, we found correlations to be smaller between older adults $(.48<r<.58)$ than between younger adults $(.58<r<.71)$.

In line with previous work (e.g., Dubossarsky et al., 2017; Wulff et al., 2018, October 29, Wulff et al., 2016), we compared the macroscopic structure of younger and older adults' networks in terms of their size $N$, average degree $\langle k\rangle$, average local clustering coefficient $C$, and average shortest path length $L$ (see Table 3). On average, older adults' networks were larger $\left(N_{O A}=5921\right.$ vs. $\left.N_{Y A}=5133\right)$, had lower degrees $\left(\langle k\rangle_{O A}=1.39\right.$ vs. $\left.\langle k\rangle_{Y A}=1.66\right)$, lower clustering coefficients $\left(C_{O A}=0.053\right.$ vs. $\left.C_{Y A}=0.115\right)$, and larger shortest path lengths $\left(L_{O A}=8.87\right.$ vs. $\left.C_{Y A}=7.45\right)$ than those of younger adults. Except for one older adult, whose network had structural characteristics more similar to those of the younger adults (Row 5 in Table 3), the same patterns were found for all possible pairwise comparisons of younger and older adults' networks. Moreover, the same patterns also emerged when we focused on the shared networks consisting of the 2,111 nodes present in all networks (Table 3).

Overall, the observed differences between younger and older adults' individual networks are well-aligned with the results of previous age-comparisons on the basis of aggregate association networks (Dubossarsky et al., 2017), aggregate verbal fluency networks (Wulff et al.,2018, October 29), and individual similarity rating networks (Wulff et al., 2018, October 29).

\section{Linking individual networks to cognitive performance}

We wanted to show the feasibility of using the networks described above to understand individual cognitive performance. Individual networks can be linked to cognitive performance at different levels. At the network level, macroscopic properties of the network, such as average degree or clustering, could be used to predict overall performance in a given task (Kenett \& Faust, 2019). At the node level, microscopic properties, such as node degree or the shortest path length between two nodes, can be used to predict trial-level performance, such as the order of retrievals in a verbal fluency task. There is value in understanding both kinds of links: Network-level links may help understand individual differences in cognitive performance that emerge globally from structural differences in semantic networks, whereas node-level links may help us understand when and how individual differences materialize for specific stimuli or contexts. The small sample size $(N=8)$ of our pilot study limits comparisons at the network-level, and therefore what follows will focus on associations of node-level links and cognitive performance. Specifically, we report how specific node characteristics estimated from our individual networks-such as node centrality, measured using PageRank, and node similarity, measured using Katz' walk similarity (De Deyne et al., 2016) — correspond to response patterns in two verbal fluency tasks, a paired associative learning task, and an episodic memory task.

In the animal and letter verbal fluency tasks, individuals were asked to retrieve as many animal words or words starting with the letter $\mathrm{S}$, respectively, as they could, within 5 minutes. The retrieved words had substantially higher centrality than words that were not retrieved. Specifically, for seven out of eight individuals, retrieved animals were more central in their respective networks as compared to other animals contained in the respective networks that were not retrieved by the individual (Figure $3 \mathrm{~A}$ ). Similarly, for all eight individuals, retrievals of words starting with the letter $\mathrm{S}$ had higher centrality in the respective networks than other words starting with any same letter contained in their networks (Figure $3 \mathrm{~B}$ ). We also found words occurring directly adjacent within retrieval sequences to be more similar to each other than words more distant from each other. Comparing directly adjacent words (Lag 1 ) to words that were three responses apart, this was the case for every individual in the animal fluency task (Figure $3 \mathrm{C}$ ) and for six of the eight individuals in the letter fluency task (Figure 3D). In the episodic memory task, participants freely recalled words from previously studied word lists. Both retrieved words and intrusions tended to have, on average, higher centrality than missing words (Figure $3 \mathrm{E}$ ). This pattern held for five out of eight individuals. Furthermore, both retrieved words and intrusions were, on average, more similar to other retrieved words than to missing words (Figure $3 \mathrm{~F}$ ) for seven of eight individuals. Finally, in the associate recall task, which required individuals to retrieve previously learned word pairs, retrieved word pairs had, on average and for all eight individuals, higher similarity than words pairs that were not retrieved (Figure 3G).

Each of these links demonstrates that individual networks can be used to predict an individual's pattern of behavior in several cognitive measures. The critical question arising from this framework, however, is whether individual networks reveal idiosyncratic differences that can be used to understand an individual's behavior better than would be possible using an aggregate representation derived from the re-

\footnotetext{
${ }^{3}$ German SWOW data was downloaded on January 25, 2021.
} 


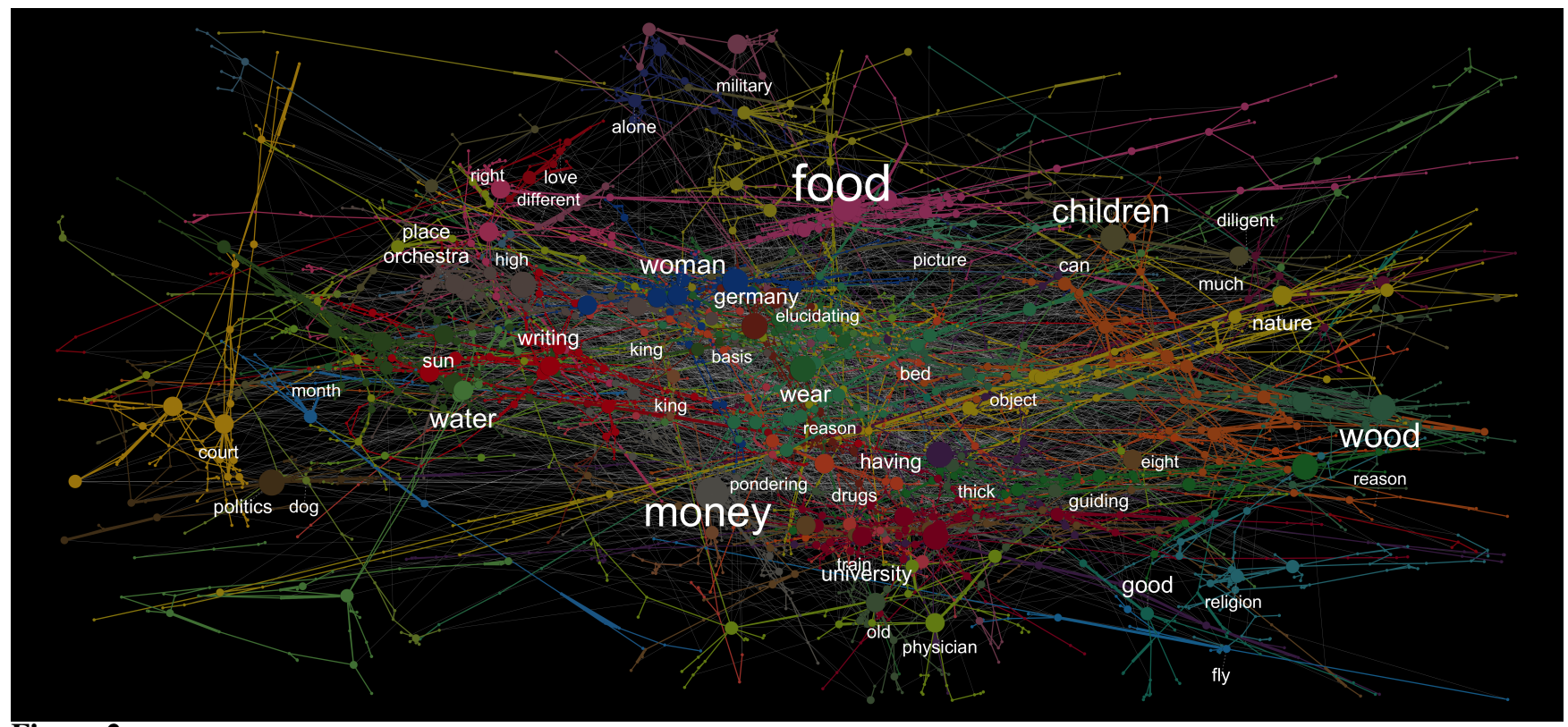

\section{Figure 2}

Associative network of one participant. Node size represents PageRank centrality, colors communities detected using the Louvain algorithm (Blondel, Guillaume, Lambiotte, $\mathcal{E}$ Lefebvre, 2008). Words were translated from the original German and show the most important words in the respective community, with size representing the word's importance in the overall network as determined by PageRank.

Table 2

Most Central (Degree) Words in Individuals' Networks

\begin{tabular}{|c|c|c|c|c|c|c|c|}
\hline \multicolumn{4}{|c|}{ Younger adults } & \multicolumn{4}{|c|}{ Older adults } \\
\hline 24 years & 27 years & 27 years & 28 years & 68 years & 68 years & 69 years & 70 years \\
\hline $\operatorname{man}$ & music & money & money & human & Germany & music & state \\
\hline music & food & write & music & country & instrument $^{1}$ & development & country \\
\hline work & work & beautiful & water & animal & military & money & goods \\
\hline change & school & food & Germany & water & car & exact & first name \\
\hline water & money & school & work & occupation & water & wood & computer \\
\hline family & clothing & read & food & work & country & make & instrument \\
\hline learn & write & important & furniture & food & food & school & family \\
\hline wood & instrument & economy & instrument & car & name & computer & university \\
\hline church & name & large & love & instrument & computer & children & church \\
\hline love & summer & family & clothes & male & child & work & month \\
\hline
\end{tabular}

Note. Words in bold face are also among the ten most central words in the German (money, music, work, school, food, water, car, love, green, important) or English (money, food, water, car, music, green, red, love, work, old) SWOW data sets. ${ }^{1}$ musical instrument

sponses of many individuals. To assess this, we compared the magnitude of the effects presented in Figure 3, e.g., the PageRank of retrieved versus non-retrieved words in verbal fluency tasks, which were derived using individuals' personal networks, against the magnitude of effects resulting from using an aggregate derived from combining the responses from all eight individuals. This analysis revealed that the aggregate network produced, on average, equally large, if not larger effects than individual networks (see black lines in Figure 3).

The similar or improved performance of the aggregate compared to individuals' own networks could be due to differences in both the reliability and precision of measurement of the different types of networks. First, on average, only .53 of three possible associations given to repeated cues were also given during the first encounter of the same cue, indicating, at best, moderate levels of internal consistency. Furthermore, focusing on the 2,111 shared nodes, we found individ- 
Table 3

Macroscropic differences in semantic network structure in terms of degree $(k)$, clustering coefficient $(C)$ and average path length $(L)$ for full networks and common networks based on nodes shared among participants.

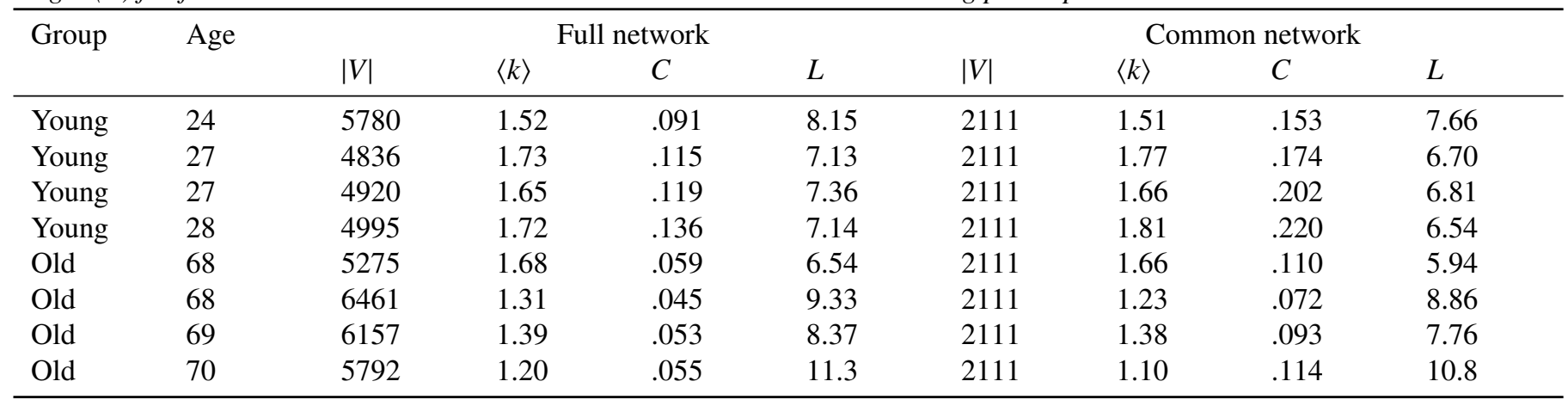

uals' networks to share considerably more variance with the aggregate, as indicated by average correlations of $\bar{r}=.61$ and $\bar{r}=.55$ for PageRank and cosine distributions, respectively, than they shared, on average, with the networks of other individuals (PageRank: $\bar{r}=.34$; Cosine: $\bar{r}=.33$ ). These results suggests that the individual networks are a noisy measurement of a shared component that is more reliably captured by an aggregate network. Second, the aggregate network is given access to more detailed information on the relative strength of associations between nodes in the networks. For individuals, only $2.2 \%$ to $5.5 \%$ of word associations occurred more than once, and these repetitions occurred a maximum of two or three times per individual. For the aggregate representation, however, $20.5 \%$ of associations occurred more than once, with counts reaching as high as 15 , leading to a more graded pattern of edge strengths between network nodes in the aggregate relative to the individual networks. One should note that this implies that the comparison of individual and aggregate networks is largely confounded with a comparison of weighted and unweighted networks.

\section{What have we learned from MySWOW?}

To summarize, our approach was successful in obtaining large-scale individual networks for a small sample of eight participants, which included both younger and older adults, suggesting that the approach can be used to capture the mental representations of individuals from several age groups.

Individuals' networks were highly similar to each other in terms of content, and these contents were qualitatively similar to those of past efforts to produce aggregate networks, such as SWOW. Nevertheless, the results from the 8 participants suggested differences in their structural composition, showing age-related differences similar to these found in past work (Dubossarsky et al., 2017, Wulff et al., 2018, October 29). Despite the small sample size, our approach seems promising in offering a potential window into adult age dif- ferences in the content and structure of the aging lexicon and the individual differences that can arise across adulthood as a function of cumulative experience. Naturally, future work needs to strive to collect data from more individuals to assess whether network-level properties are systematically related to differences in cognitive performance between individuals.

Our results also showed that estimates of node centrality and relatedness derived from single individuals' networks exhibited strong links to individuals' responses in four cognitive tasks, including two verbal fluency and two memory tasks. These results demonstrate that it is feasible, in principle, to map information from individual semantic networks onto additional linguistic and memory performance at the single participant level.

Our results demonstrate that our approach is, nevertheless, limited. Most crucially, our results suggest that individual networks were less powerful in accounting for individuals' response patterns in the additional measures of linguistic and memory performance than an aggregate network composed of all individuals' responses. The advantage of aggregate over individual networks presents a major challenge to the claim that it is important to assess individual networks to understand individual differences in cognitive performance. Before one fully dismisses our claim, one should distinguish two explanations for these findings that have different implications. First, one possibility is that our approach was not able to capture relevant individual differences in the content of the semantic representations, for example, because of the use of a pool of cues containing mostly high-frequency words for which individual differences in semantic representations may be small. In this case, the breadth of cues used would need to be expanded so that the networks generated are better able to capture the idiosyncratic nature of individuals' representations. Second, another possibility is that our approach may not have been able to measure the strength of association between various nodes (i.e., edges) with sufficient precision and reliability. In this case, additional work 

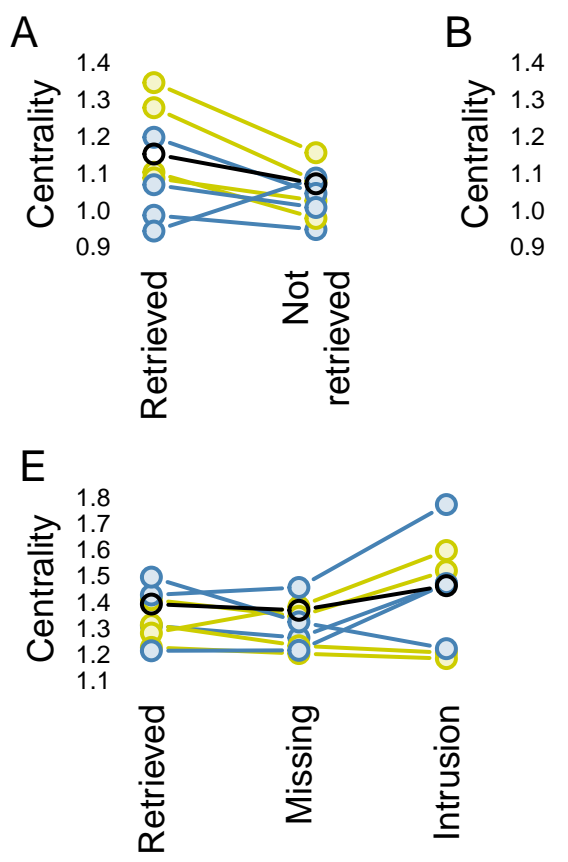
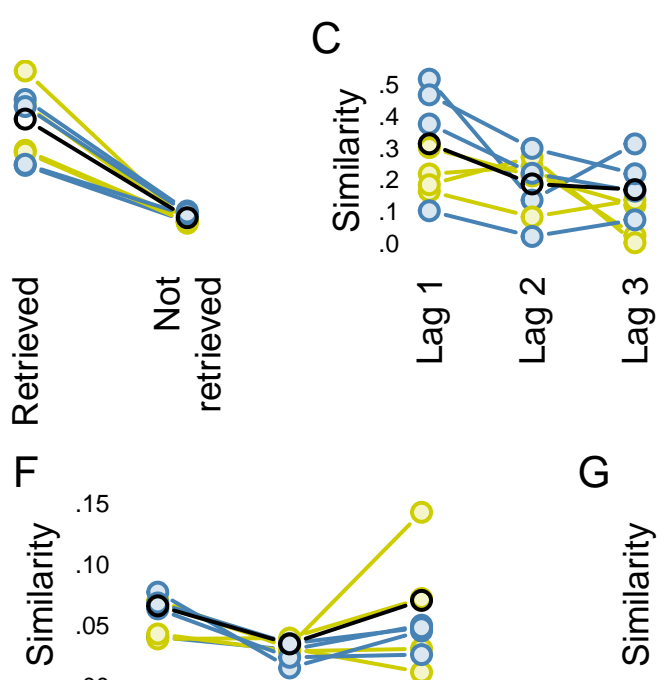

.00
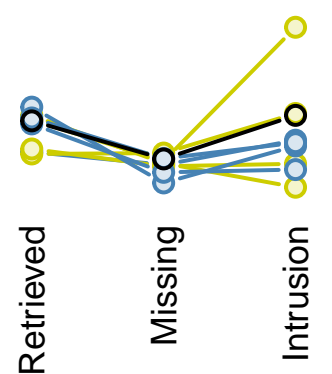

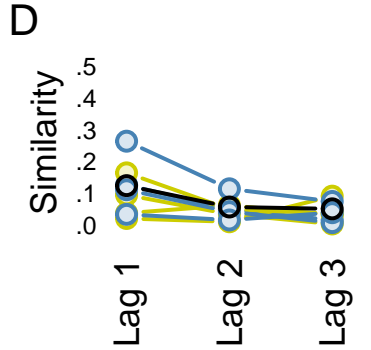

G

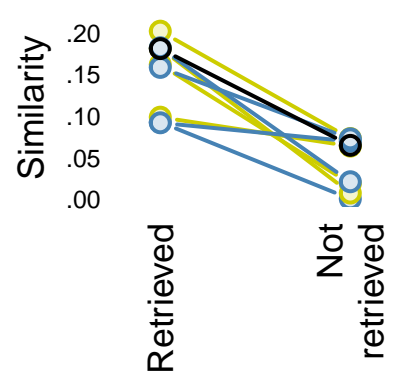

\section{Figure 3}

Links between mental representations and cognitive performance for the four younger (blue lines) and the four older adults (yellow lines). The panels show the difference in PageRank centrality between retrieved and non-retrieved words in animal $(A)$ and letter fluency $(B)$, the average cosine similarity of words one, two, and three words apart in animal (C) and letter fluency (D), the PageRank centrality of retrieved, missing, and intrusion words in the episodic memory task (E), the average cosine similarity of retrieved, missing, and intrusion words to (other) retrieved words in the episodic memory task $(F)$, and the average cosine similarity of retrieved versus non-retrieved word pairs in the associative recall task $(G)$. The black line shows the effects under the aggregate representaions. Centrality is measured as PageRank times the number of nodes in the nodes in the network, to account for differences in network sizes.

may be needed that considers both obtaining larger sets of responses per cue, having participants provide responses to the same cue on repeated occasions, or asking individuals to provide additional ratings (Roads \& Love, 2020). In our view, these possibilities speak to the need for future work that guarantees appropriate scope and reliability rather than a clear rejection of the idea that individual networks can be helpful to understanding the link between experience and cognitive performance.

\section{General Discussion}

Older adults tend to perform worse on a broad set of cognitive tasks, and such findings are commonly attributed to a decline in fluid cognitive abilities (Healey \& Kahana, 2016, Salthouse, 2010). However, some have argued that changes in the underlying size and structure of representations can contribute to age differences in cognitive performance, for example, due to activation-spreading across many targets in memory (i.e., fan effect; Buchler and Reder, 2007) or difficulties in discrimination learning between many similar items (Ramscar et al., 2017), which can be a direct conse- quence of individuals' cumulative interaction with the physical, linguistic, and social world. In this paper, we proposed an empirical agenda to provide an estimate of the extent to which idiosyncratic experiences matter for the size and structure of mental representations and, ultimately, individual and age differences in cognitive performance. Despite the promise we see in this approach, a number of other, major challenges remain.

First, our suggested approach of mapping individual semantic networks is open to the criticism that no elicitation of individuals' knowledge store is independent of the process by which the representation is accessed. As a result, any individual or age differences detected cannot be unequivocally assigned to the nature of association in the knowledge store (representation), but can in principle result from the process by which this representation is searched and accessed (Jones, Hills, et al., 2015, Kenett et al., 2020, Kraemer et al., 2021, February 10; Siew et al., 2019). So far, there seems to be no clear consensus concerning the extent to which representation and process are entangled in the kinds of tasks presented here (Abbott et al., 2015; Jones, Hills, et al., 2015). Recent studies have addressed this question empirically and found 
that the retrieval processes might underestimate semantic effects due to frequency biases, which need be mitigated using appropriate transformations to associative strength measures (De Deyne et al., 2019). More generally, it is unlikely that a single approach can provide a definitive resolution to this conundrum and there are two possible ways forward that we would like to emphasize. One potential way to distinguish the role of structure versus process could be to use multiple methods of assessment (e.g., word association, verbal fluency, relatedness judgments) to elicit mental representations. To the extent that these different assessment methods are associated with different search processes and strategies, convergent evidence could provide some support for the role of representation. A related approach could involve the use of the same elicitation method under different explicitly instructed search strategies that could equate these across participants (Wulff et al., 2020, January 16; Wulff et al., 2013). A second direction could involve using neuroscience techniques to distinguish between search (i.e., control) processes and representational components (Hoffman \& Morcom, 2018), in particular to the extent that individuals' representational space has a signature in the functional organization of the brain (Huth et al., 2012).

Second, our approach proposed a dedicated experimental procedure (i.e., word-association task) to capture the mental representations of single individuals. However, there may be more efficient and powerful alternatives that should be explored, for example massive recording and analysis of naturalistic stimuli and behavior, including both linguistic sources such as speech (Mehl, 2017) and text production (Banda et al., 2020), and non-linguistic ones such as sensorial input (Sadeghi et al., 2015). These approaches could become particularly powerful as such data become increasingly connected with other information about individuals, for example, if these are collected with informed consent in larger surveys or longitudinal household panels.

Third, the field has seen considerable progress in the computational modeling of the acquisition of mental representations (Jones, Willits, et al., 2015) as well as search (Abbott et al., 2015). However, neither we nor others have used such models to systematically investigate individual differences. Ideally, the deployment of large-scale empirical efforts as the one we discussed above will be preceded by simulation and modeling work that can provide a guide concerning which structures and magnitudes of differences are most likely to be expected and captured with which cognitive paradigm.

Fourth, and finally, most of the research discussed above (including our own approach) is correlational in nature, in that it aims to establish a correlation between individual experience and the contents and structure of mental representation or cognitive performance. In order to help establish causality, however, one would need a comparison of individuals assigned to different environmental exposures through either natural or, ideally, controlled experiments. There are a few candidate training strategies, including training of specific physical or virtual environments (Miller et al., 2013), artificial or natural languages (Pothos, 2007), and even complex narratives (Heusser et al., 2021)). Regardless of the exact type of information and mode of exposure, it remains a challenge to obtain meaningful and reliable individual estimates of the emergent mental representations and their potential effects across the life span. We hope our review of past work and our own proposal contribute to this larger effort.

\section{Conclusion}

We have argued that quantifying individual and age differences in the size and structure of human knowledge is important because this represents a missing link in estimating the role of cumulative experience in cognitive performance. We specifically proposed an empirical agenda that combines tracking individuals' idiosyncratic experiences and broad mapping of their mental representations and cognitive performance. We hope such steps can move us closer to understanding the role of the environment in the mental lexicon and its implications for cognitive aging.

\section{References}

Abbott, J. T., Austerweil, J. L., \& Griffiths, T. L. (2015). Random walks on semantic networks can resemble optimal foraging. Psychological Review.

Banda, J. M., Tekumalla, R., Wang, G., Yu, J., Liu, T., Ding, Y., Artemova, K., Tutubalina, E., \& Chowell, G. (2020). A large-scale covid-19 twitter chatter dataset for open scientific research - an international collaboration.

Benedek, M., Kenett, Y. N., Umdasch, K., Anaki, D., Faust, M., \& Neubauer, A. C. (2017). How semantic memory structure and intelligence contribute to creative thought: A network science approach. Thinking $\mathcal{E}$ Reasoning, 23(2), 158-183.

Blondel, V. D., Guillaume, J.-L., Lambiotte, R., \& Lefebvre, E. (2008). Fast unfolding of communities in large networks. Journal of statistical mechanics: theory and experiment, 2008(10), P10008.

Brysbaert, M., Stevens, M., Mandera, P., \& Keuleers, E. (2016). How Many Words Do We Know? Practical Estimates of Vocabulary Size Dependent on Word Definition, the Degree of Language Input and the Participant's Age. Frontiers in psychology, 7(021006), 55-11.

Buchler, N. E. G., \& Reder, L. M. (2007). Modeling agerelated memory deficits: A two-parameter solution. Psychology and aging, 22(1), 104-121. 
De Deyne, S., Navarro, D. J., Perfors, A., \& Storms, G. (2016). Structure at every scale: A semantic network account of the similarities between unrelated concepts. Journal of Experimental Psychology: General, 145(9), 1228.

De Deyne, S., Navarro, D. J., \& Storms, G. (2012). Better explanations of lexical and semantic cognition using networks derived from continued rather than single-word associations. Behavior Research Methods, 45(2), 480-498.

De Deyne, S., Navarro, D. J., Collell, G., \& Perfors, A. (2021). Visual and affective multimodal models of word meaning in language and mind. Cognitive Science, 45(1), e12922.

De Deyne, S., Navarro, D. J., Perfors, A., Brysbaert, M., \& Storms, G. (2018). The "Small World of Words" English word association norms for over 12,000 cue words, 1-20.

De Deyne, S., Navarro, D. J., Perfors, A., Brysbaert, M., \& Storms, G. (2019). The "small world of words" english word association norms for over 12,000 cue words. Behavior research methods, 51(3), 9871006.

De Deyne, S., \& Storms, G. (2008). Word associations: Norms for 1,424 dutch words in a continuous task. Behavior research methods, 40(1), 198-205.

Dubossarsky, H., De Deyne, S., \& Hills, T. T. (2017). Quantifying the structure of free association networks across the life span. Developmental psychology, 53(8), 1560.

Gobet, F., \& Simon, H. A. (1996). Recall of random and distorted chess positions: Implications for the theory of expertise. Memory $\mathcal{E}$ cognition, 24(4), 493-503.

Healey, M. K., \& Kahana, M. J. (2016). A four-component model of age-related memory change. Psychological Review, 123(1), 23.

Heusser, A. C., Fitzpatrick, P. C., \& Manning, J. R. (2021). Geometric models reveal behavioural and neural signatures of transforming experiences into memories. Nature Human Behaviour, 1-26.

Hoffman, P., \& Morcom, A. M. (2018). Age-related changes in the neural networks supporting semantic cognition: A meta-analysis of 47 functional neuroimaging studies. Neuroscience $\mathcal{E}$ Biobehavioral Reviews, 84, 134-150.

Huettig, F., \& Pickering, M. J. (2019). Literacy Advantages Beyond Reading: Prediction of Spoken Language. Trends in Cognitive Sciences, 23(6), 464-475.

Huth, A. G., Nishimoto, S., Vu, A. T., \& Gallant, J. L. (2012). A Continuous Semantic Space Describes the Representation of Thousands of Object and Action Categories across the Human Brain. Neuron, 76(6), 1210-1224.
Jones, M. N., Willits, J., \& Dennis, S. (2015). Models of semantic memory. The Oxford Handbook of ...

Jones, M. N., Hills, T. T., \& Todd, P. M. (2015). Hidden processes in structural representations: A reply to abbott, austerweil, and griffiths (2015).

Kenett, Y. N., Beckage, N. M., Siew, C. S., \& Wulff, D. U. (2020). Cognitive network science: A new frontier. Complexity.

Kenett, Y. N., \& Faust, M. (2019). A Semantic Network Cartography of the Creative Mind. Trends in Cognitive Sciences, 23(4), 271-274.

Kraemer, P. M., Wulff, D. U., \& Gluth, S. (2021, February 10). A sequential sampling account of semantic relatedness decisions. https://doi.org/10.31234/osf.io/ ksa2g

Li, H., N, K. Y., Kaixiang, Z., Cheng, L., Rongcan, Z., Tingrui, Y., Tengbin, H., \& Jiang, Q. (2020). The relation between semantic memory structure, associative abilities, and verbal and figural creativity. Thinking $\mathcal{F}$ Reasoning, $0(0), 1-26$.

Lindenberger, U. (2014). Human cognitive aging: corriger la fortune? Science, 346(6209), 572-578.

Love, R., Dembry, C., Hardie, A., Brezina, V., \& McEnery, T. (2017). The spoken bnc2014: Designing and building a spoken corpus of everyday conversations. International Journal of Corpus Linguistics, 22(3), 319-344.

Mehl, M. R. (2017). The electronically activated recorder (ear): A method for the naturalistic observation of daily social behavior [PMID: 28529411]. Current Directions in Psychological Science, 26(2), 184190. https://doi.org/10.1177/0963721416680611

Miller, J. F., Neufang, M., Solway, A., Brandt, A., Trippel, M., Mader, I., Hefft, S., Merkow, M., Polyn, S. M., Jacobs, J., Kahana, M. J., \& Schulze-Bonhage, A. (2013). Neural Activity in Human Hippocampal Formation Reveals the Spatial Context of Retrieved Memories. Science, 342(6162), 1111-1114.

Mol, S. E., \& Bus, A. G. (2011). To Read or Not to Read: A Meta-Analysis of Print Exposure From Infancy to Early Adulthood. Psychological Bulletin, 137(2), 267-296.

Morais, A. S., Olsson, H., \& Schooler, L. J. (2013). Mapping the structure of semantic memory. Cognitive science, 37(1), 125-145.

Nation, K. (2017). Nurturing a lexical legacy: reading experience is critical for the development of word reading skill. NPJ science of learning, 1-.

Nelson, D. L., Bennett, D. J., Gee, N. R., Schreiber, T. A., \& McKinney, V. M. (1993). Implicit memory: Effects of network size and interconnectivity on cued recall. Journal of experimental psychology. Learning, memory, and cognition, 19(4), 747-64. 
Pothos, E. M. (2007). Theories of artificial grammar learning. Psychological Bulletin, 133(2), 227-244.

Ramscar, M., Hendrix, P., Shaoul, C., Milin, P., \& Baayen, H. (2014). The myth of cognitive decline: Non-linear dynamics of lifelong learning. Topics in cognitive science, 6(1), 5-42.

Ramscar, M., Sun, C. C., Hendrix, P., \& Baayen, H. (2017). The mismeasurement of mind: Life-span changes in paired-associate-learning scores reflect the "cost" of learning, not cognitive decline. Psychological science, 28(8), 1171-1179.

Roads, B. D., \& Love, B. C. (2020). Enriching imagenet with human similarity judgments and psychological embeddings. arXiv preprint arXiv:2011.11015.

Rodd, J. M. (2020). Settling Into Semantic Space: An Ambiguity-Focused Account of Word-Meaning Access. Perspectives on Psychological Science, 174569161988586-17.

Sadeghi, Z., McClelland, J. L., \& Hoffman, P. (2015). You shall know an object by the company it keeps: An investigation of semantic representations derived from object co-occurrence in visual scenes. $\mathrm{Neu}$ ropsychologia, 76(3), 52-61.

Sala, G., \& Gobet, F. (2017). Experts' memory superiority for domain-specific random material generalizes across fields of expertise: A meta-analysis, 1-11.

Salthouse, T. A. (2010). Selective review of cognitive aging. Journal of the International neuropsychological Society, 16(5), 754-760.

Schröter, P., \& Schroeder, S. (2017). The developmental lexicon project: A behavioral database to investigate visual word recognition across the lifespan. Behavior Research Methods, 49(6), 2183-2203.

Siew, C. S., Wulff, D. U., Beckage, N. M., \& Kenett, Y. N. (2019). Cognitive network science: A review of research on cognition through the lens of network representations, processes, and dynamics. Complexity, 2019.
Steyvers, M., Shiffrin, R. M., \& Nelson, D. L. (2004). Word association spaces for predicting semantic similarity effects in episodic memory. Experimental cognitive psychology and its applications: Festschrift in honor of Lyle Bourne, Walter Kintsch, and Thomas Landauer, 237-249.

Twenge, J. M., Martin, G. N., \& Spitzberg, B. H. (2019). Trends in U.S. Adolescents' media use, 1976-2016: The rise of digital media, the decline of TV, and the (near) demise of print. Psychology of Popular Media Culture, 8(4), 329-345.

Verhaeghen, P. (2003). Aging and vocabulary scores: A meta-analysis. Psychology and aging, 18(2), 332-9.

Wulff, D. U., De Deyne, S., Jones, M. N., Mata, R., \& Aging Lexicon Consortium. (2019). New Perspectives on the Aging Lexicon. Trends in Cognitive Sciences, 23(8), 686-698.

Wulff, D. U., Hills, T., \& Hertwig, R. (2020, January 16). Memory is one representation not many: Evidence against wormholes in memory. https://doi.org/10. 31234/osf.io/b5ynj

Wulff, D. U., Hills, T., \& Mata, R. (2018, October 29). Structural differences in the semantic networks of younger and older adults. https://doi.org/10.31234/ osf.io/s73dp

Wulff, D. U., Hills, T. T., \& Hertwig, R. (2013). Worm holes in memory: Is memory one representation or many? Proceedings of the Annual Meeting of the Cognitive Science Society, 35(35).

Wulff, D. U., Hills, T. T., Lachman, M., \& Mata, R. (2016). The aging lexicon: Differences in the semantic networks of younger and older adults. Proceedings of the Annual Meeting of the Cognitive Science Society, 38(38).

Zemla, J. C., \& Austerweil, J. L. (2018). Estimating semantic networks of groups and individuals from fluency data. Computational Brain $\mathcal{F}$ Behavior, 1-23. 